\title{
Importance of mediastinoscopy, bilateral thoracoscopy, and laparoscopy in correct staging of malignant mesothelioma before extrapleural pneumonectomy
}

\author{
J. M. Alvarez, FRACS, ${ }^{a}$ T. Ha, MBBS, ${ }^{a}$ W. Musk, FRACP, ${ }^{\text {b }}$ P. Robins, FRACP, ${ }^{\mathrm{c}}$ R. Price, FRACR, FRANZCR, ${ }^{d}$
}

and M. J. Byrne, FRACP, e Perth, Australia

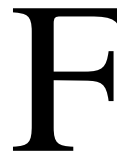
or selected patients with malignant mesothelioma, treatment with extrapleural pneumonectomy (EPP), chemotherapy, and hemithoracic irradiation (trimodality therapy) appears to confer improved survival relative to other treatment options. ${ }^{1}$ Sugarbaker and colleagues ${ }^{1}$ demonstrated a 5 -year survival of $46 \%$, with a median survival of 51 months, for patients with epithelial histologic type, negative extrapleural nodes, and negative resection margins. We therefore offer trimodality therapy to selected patients with stage 1 (limited to one hemithorax) epithelial malignant mesothelioma. All patients have preoperative computed tomographic (CT), magnetic resonance imaging (MRI), and positron emission tomographic (PET) scans. ${ }^{1,2}$ However, EPP is only offered to those with histologically confirmed negative results of biopsy examinations of the mediastinum, contralateral pleura, and diaphragmatic peritoneum.

\section{Clinical Summary}

A 67-year-old woman was incidentally discovered to have a right pleural effusion. Cytologic examination confirmed malignant mesothelioma. CT, MRI, and PET scans (Figure 1, A-C), demonstrated stage 1 disease. The patient met established criteria for trimodality therapy. ${ }^{1}$

Our staging protocol included mediastinoscopy, bilateral thoracoscopy, and laparoscopy, with respective biopsy samples. Mediastinoscopy revealed normal nodes. A left thoracoscopic sample was macroscopically normal; random specimens were taken from three midaxillary costal sites: superior, midzone, and inferior. Laparoscopy revealed a 3-mm wide, gray, flat nodule on the dome of the right diaphragm. Right thoracoscopy confirmed extensive malignant mesothelioma involving parietal and visceral pleura. Histopathologic examination revealed normal mediastinal nodes. Epithelial malignant mesothelioma was present in the right pleural, all left pleural (Figure $1, D)$, and the diaphragmatic biopsy specimens. Cytologic examination of peritoneal lavage fluid yielded negative results. The disease

From the Departments of Cardiothoracic Surgery, ${ }^{\text {a }}$ Respiratory Medicine, ${ }^{\mathrm{b}}$ Nuclear Medicine, ${ }^{\mathrm{c}}$ Radiology, ${ }^{\mathrm{d}}$ and Oncology, ${ }^{\mathrm{e}}$ Sir Charles Gairdner Hospital, Perth, Australia.

Received for publication Dec 22, 2004; accepted for publication Feb 8, 2005.

Address for reprints: J. M. Alvarez, FRACS, Department of Cardiothoracic Surgery, Sir Charles Gairdner Hospital, Nedlands 6009, Perth, West Australia, Australia.

J Thorac Cardiovasc Surg 2005;130:905-6

$0022-5223 / \$ 30.00$

Copyright $\odot 2005$ by The American Association for Thoracic Surgery

doi:10.1016/j.jtcvs.2005.02.051 was therefore restaged as stage 3 , and the patient was offered chemotherapy.

\section{Discussion}

The optimal management of stage 1 malignant mesothelioma is unclear. EPP is an operation associated with significant mortality $(10 \%-30 \%)$ and morbidity. Sugarbaker and colleagues ${ }^{2}$ have reduced perioperative mortality for EPP to $5 \%$ with trimodality therapy and have reported unequalled survival in selected cases of malignant mesothelioma. ${ }^{2}$

Patterns of tumor recurrence after trimodality therapy reveal that $26 \%$ recur in the peritoneum. Conventional wisdom suggests the mechanism may relate to peritoneal seeding during surgery. However, $17 \%$ of malignant mesothelioma recurrences are in the contralateral pleura, and importantly, distant metastases are rare. ${ }^{3}$ This suggests that preoperative understaging may actually have occurred. Because of the significant morbidity associated with this radical protocol, the finite sensitivities of current imaging modalities, and the intimate $(<1 \mathrm{~mm})$ anatomic relations of both parietal pleura, the mediastinum, and peritoneum, we advocate bilateral thoracoscopy, mediastinoscopy, and laparoscopy, with respective biopsy samples, as integral to correctly staging malignant mesothelioma.

This case is important because it emphasizes that surgical preoperative staging appears necessary to prevent radical treatment of patients who would not actually benefit and that current nonsurgical staging procedures are not yet sufficiently sensitive to replace surgical staging. The sensitivity of noninvasive imaging is limited in low-volume or micrometastatic disease. State-of-the-art multislice CT, MRI, and PET scans were unable to detect transdiaphragmatic or contralateral pleural disease, and the pleura even appeared normal on visual inspection at thoracoscopy.

We offer trimodality therapy only to patients with epithelial histologic type. Surgical pleural biopsy is thus needed. Unilateral thoracoscopy, mediastinoscopy, and laparoscopy and contralateral thoracoscopy are performed. Operating time approaches 1 hour. Potential morbidity from these additional procedures appears justified by the profound impact that a positive result confers on treatment and prognosis. Our findings are important not only to centers offering trimodality therapy but also for staging malignant mesothelioma in general. Incorrect staging will invalidate trimodality therapy and subject patients to unnecessary, painful, and potentially harmful complications. Correct staging can only be achieved by a protocol that includes mediastinoscopy, laparoscopy, and bilateral thoracoscopy. 

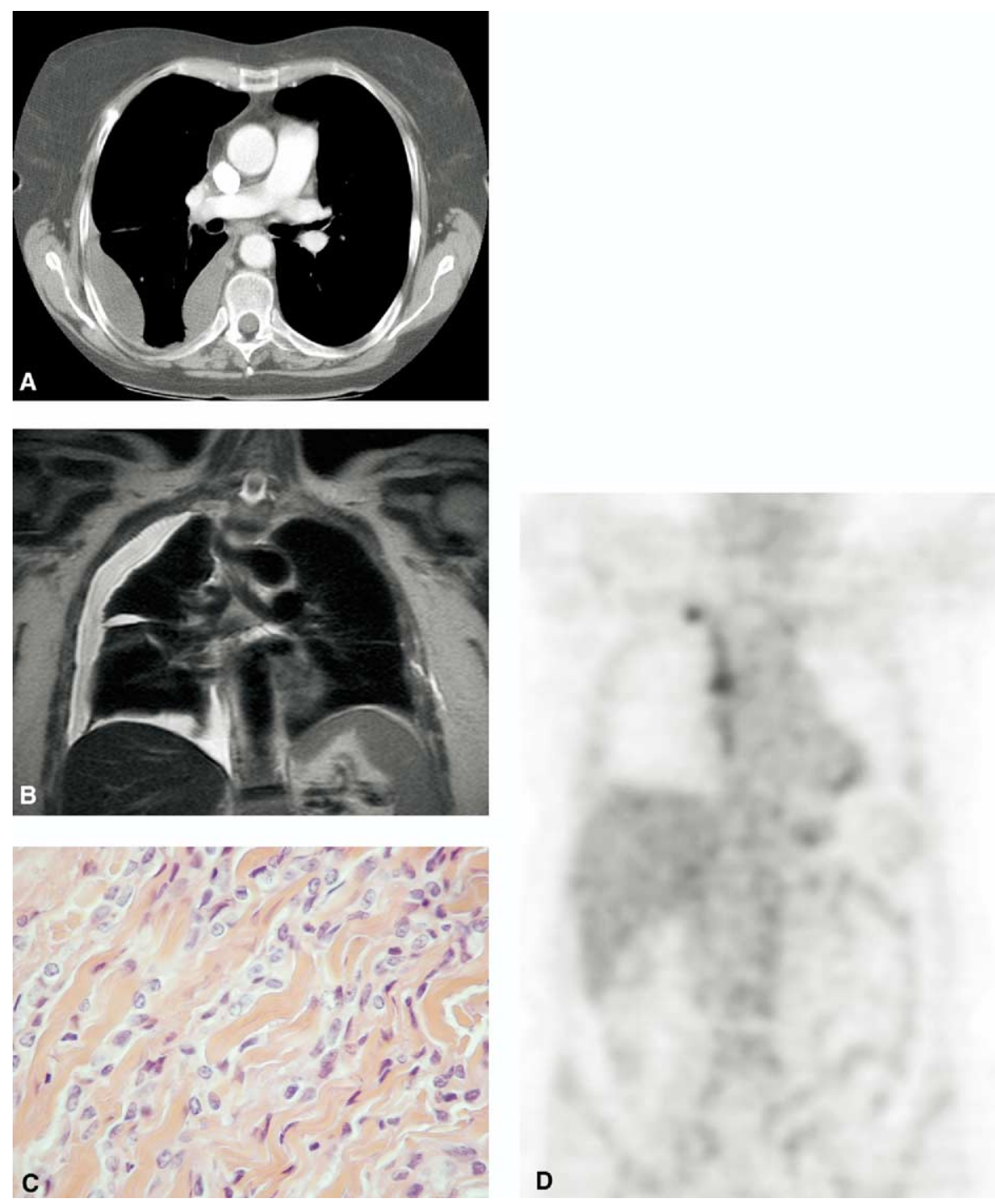

Figure 1. A, CT scan. Axial postcontrast image at the level of the pulmonary trunk bifurcation. Right-sided pleural effusion is present without discernible pleural mass. Left pleural surface is unremarkable. B, MRI scan. Coronal $\mathrm{T}_{2}$-weighted half-Fourier acquisition single-shot turbo spin-echo (HASTE) through the carina. Right-sided pleural effusion is present; no discernible pleural mass is evident. Left pleura is normal. C, PET scan. Intense uptake along the right mediastinal pleura and right-sided effusion. The left pleura appears normal. D, Thin ribbons of malignant mesothelioma cells infiltrating between layers of collagen; in addition, surface mesothelial cells (not shown) show patchy foci of membranous staining to epithelial membrane antigen within enlarged, atypical mesothelial cells.

\section{References}

1. Sugarbaker D, Flores RM, Jaklitsch MT, Richards WG, Strauss GM, Corson JM, et al. Resection margins, extrapleural nodal status and cell type determine postoperative long-term survival in trimodality therapy of malignant mesothelioma: results in 183 patients. J Thorac Cardiovasc Surg. 1999;117:54-65.
2. Sugarbaker D, Jaklitsch MT, Bueno R, Richards W, Lukanich J, Mentzer SJ, et al. Prevention, detection and management of complications after 328 consecutive extrapleural pneumonectomies. J Thorac Cardiovasc Surg. 2004;128:138-46.

3. Baldini EH, Recht A, Strauss GM, DeCamp MM Jr, Swanson SJ, Liptay MJ, et al. Patterns of failure after trimodality therapy for malignant mesothelioma. Ann Thorac Surg. 1997;63:334-8. 